\title{
Community Engagement and the Creation of Social Capital
}

\author{
Muhammad Adha Shaleh*
}

Since the concept of sustainable development gained traction in the early $70 \mathrm{~s}$, there has been demand for new approaches, perspectives, and practices to the conservation of natural resources. The proposition that much of our modern environmental policies need citizen participation is widely accepted. In line with this standpoint, several concepts have emerged. They are shaped by FaithBased Environmental Conservation, theoretical developments on environmental governance of the commons (e.g. Community Based Natural Resources Management), and social capital. The first and the second concepts indicate the integration of religious principles and social ecological wisdom into natural resources management. The third concept captures the idea that social bonds, norms, cultures, knowledge, attitudes, and information channels are central for a sustainable environment. In a highly organised group, where social capital is strong, the pace towards environmental sustainability has been expedited by successes in community-based programmes.

However, that generalisation of 'good cases' often does not point out a practical grounding explaining what makes social capital so successful. Recent years have shown the pivot of community engagement as being an effective route to the protection of natural resources. This macro-level phenomenon is considered by field-based researchers as a prelude to the creation of social capital. It draws public participation and collective interventions in the planning and designing of environmental management plans and activities. Much of its focus is investing in collective actions, as people cooperate at localities, for a better structure to guard common resources. In relation to this point, there is interest about community roles in environmental protection, which has reached a new plateau in Malaysia. As interest surged in recent years, fresh perspectives on community-centred activities have emerged, and collaborative partnership between the state and local communities have been lubricated. One case study in Terengganu saw a collaboration between NGOs and religious departments that produced Friday sermons carrying the wildlife conservation theme. In this manner, the concept of community engagement offers an idea: to start the initial process of creating, piloting, and developing a small structure for a better environmental care. In other words, it offers a realistic step by step grounding towards the implementation of large scale conservation projects. 
There is, however, one key issue that can be seen as a barrier to initiating community engagement activities - reluctance to engage in short-term projects. This issue is hard to refute. The compelling logic is that citizens and communities perceive short-term and time-specific projects as unsustainable, lacking the continuity to benefit both people and their environment. Most of these sorts of projects demotivated people to commit actively as they make no long-term benefits to them. Another excuse for non-participation is 'the long standing focus' on state regulations and market-based policies, without the long run strategies for community engagement efforts.

However, as our urbanisation, population and modernisation have increased, and as degradation, exploitation and pollution have accelerated, the responses seem starker. Citizens and communities are bound either to wait for top-down regulations to prevent further damages or they engage. The latter is, in principle, an important and a highly vital catalyst especially to the implementation of sustainable natural resource management. Furthermore, many field-based studies that were carried out in small scale societies worldwide had provided background for an innovative concept of community engagement. The studies consistently recognised that it is easy to increase social capital because valued relation promotes peoples' compliance with rules and keeps down monitoring costs. Also, with frequent communication and reciprocal arrangements between group members, and with small group size structure, members of the society can manage natural resources collectively. As a result of these concerted community engagement efforts, there has been an increasing number of cases that show highlevel bio-diversities and biological growth in local areas.

Although we have argued that community engagement activities potentially benefit community-led environmental projects, this idealistic vision may not be the panacea. Despite its validity in today's pressing environmental issues, and despite many implicit case studies inciting us to believe in its environmental and social payoff, it seems to focus on the net impact of social capital. We should not be too optimistic about its popularity and accuracy. Rather we should remember that there is a higher level of concern, and that is to help a nation's stock of social capital through community engagement. Thus, this concern leads us to stress the value of environmental knowledge, the value of effective institutions, and the need for adequate funding for community-based projects. A recognition of the following three external catalysts is required to reach the wider goal of community engagement.

First, citizens/communities may not always have the knowledge to anticipate that what they are doing may be harmful to the ecosystem. Thus, there is a need for them to collaborate with scientists who hold the trove of knowledge for a better implementation of conservation projects. 
Second, citizens may not always correctly predict climate change, and they do not have the power to restrain the pressures of national, regional, and global markets. These are partly the reasons for their reluctance to engage in conservation projects. The logic is simple: no community believes it can have a big impact on national, regional and global phenomena. Thus, effective higher level institutions are needed to complement local institutional arrangements.

The third value offers a more pragmatic vision for a long and sustainable community engagement project. With enough funding from government or the private sector, it leads to a favourable cooperation by community members, for the protection of the environment, both in the short and in the longer terms. State support for community-based projects can assist in facilitation. In turn, there are chances of bridging divides, building trust and developing mutual understanding between communities and other stakeholders.

This viewpoint signals some important directions for the vitality of community engagement in the creation of social capital. In general, the participation of citizens in any environmental project, and in resources care plans, is handicapped by the lack of proper facilitation, environmental knowledge and financial support. But, if these challenges are properly addressed, community engagement in environmental programmes can salvage nation's reputation, as well as build public credibility, trust and confidence.

To conclude, we have seen that community engagement approaches corroborate citizen participation in resource care. Much of its concern is to catalyse the formation of sustainable resources care. It seems to effectively initiate low cost environmental governance, and perhaps can offer a quick route to a better environmental outcome in the future. To date, it has affected the levels of social, cultural and natural capital worldwide. Such growth - both in academic discourses, and actual implementations - is certainly worth a closer look.

* Muhammad Adha Shaleh is a Research Fellow at the International Institute of Advanced Islamic Studies (IAIS) Malaysia. He can be contacted at adha@iais. org.my. 